\title{
Editorial: Biotribology and Biotribocorrosion Properties of Implantable Biomaterials
}

\author{
Alessandro Ruggiero ${ }^{1 *}$ and Hongyu Zhang ${ }^{2}$ \\ ${ }^{1}$ Department of Industrial Engineering, University of Salerno, Salerno, Italy, ${ }^{2}$ State Key Laboratory of Tribology, Department of \\ Mechanical Engineering, Tsinghua University, Beijing, China
}

Keywords: biotribology, biotribocorrosion, biomaterials, surface modification, in-silico models, biomechanical models

\section{Editorial on the Research Topic}

\section{Biotribology and Biotribocorrosion Properties of Implantable Biomaterials}

This special issue was conceived with the aim to give to the readers the opportunity to focus their attention on some recent and relevant scientific issues in the field of Biotribology and Biotribocorrosion, which represents nowadays one of the most interesting areas of Tribology applied to the study of biological systems. The word "biotribology" was introduced by Dowson in 1970 as "those aspects of tribology concerned with biological systems" (Dowson, 1970; Zhongmin et al., 2020) and represents a fascinating multidisciplinary Research Topic, focused on the study of friction, wear and lubrication of natural and artificial synovial joint, wear of dentures, ocular tribology, wear of cardiac valves and lubrication of artificial heart pumps, tribology of artificial screws and plates against natural bone, comfort of clothes, tribology for assessing mouthfeel attributes of foods, etc. (Jin et al., 2006). When the combined effects of wear and corrosion due to the biological environments are considered, biotribocorrosion takes place. In this scenario the biotribology and the biotribocorrosion properties of modern implantable biomaterials are extremely important, especially for the situations where there is relative movement between the implanted biomaterials or between the implanted biomaterial and the natural tissues under physiological environment. This is the case for example of the Total Knee Replacements (TKR) or Total Hip Replacements (THR) which represent one of the most investigated bio-tribological system due to their growing diffusion in all the world (Kurtz, et al., 2007; Learmonth et al., 2007). In TKR and THR wear phenomena play a key role, since one of the main failure reasons are dislocation due to debris particles entrapped in human tissues leading to metallosis and osteolysis conditions (Kaddick et al., 2009; Zivic et al., 2017). With the aim to improve the tribological design of such components, preclinical in-vitro wear tests by knee and hip simulators has become a standard procedure for achieving a performance assessment and for investigating experimentally the tribological phenomena acting on the contact surfaces during a simulated motion in a controlled lubricating environment (Abdel-Jaber et al., 2015). However, these tests are expensive and require long time, since the simulations run for several million cycles, taking in to account that one million cycles is assumed to correspond to 1 year in-vivo conditions. Considering the above, to date, in-silico wear prediction models of artificial human implants attract the attentions of researchers to obtain complete tribological theoretical and numerical models useful for the in-silico testing (O'Brien et al., 2015; Mattei et al., 2016; Affatato et al., 2018), which could avoid the standard in-vitro time-consuming investigation procedures (simulators) and could contribute as tool for a more and more accurate tribological design of human prostheses. Obviously, the accurate wear prediction of artificial joints requires to develop detailed tribological models accounting for 
the complexity and the multiscale of wear phenomenon (Vakis et al., 2018) which requires scientific knowledge in many fields, such as contact mechanics (Popov, 2010), topographic contact surfaces characterization (Merola et al., 2016), new materials formulations (Affatato et al., 2015), stress-strain analysis and FEM/BEM simulations (Ruggiero et al., 2018; Ruggiero and D'Amato R, 2019), musculoskeletal multibody modeling (Zhang et al., 2017), unsteady synovial lubrication modeling (boundary/mixed, hydro-dynamic and EHD) (Ruggiero and Sicilia, 2020), tribo-corrosion (Tan et al., 2016), metal transfer phenomena (Affatato et al., 2017), biomaterials characterizations (Ruggiero et al., 2016), etc. Moreover, innovative biomaterials and manufacturing procedures (e.g., 3D printing), novel surface modification (coatings) constitute new and exciting research areas (Ten Kate et al., 2017).

In this framework this special issue aims to inspire colleagues and scientists collecting interesting research papers investigating the THR wear of CoCrMo femoral heads, against conventional and X-linked ultra-high molecular weight polyethylene (UHMWPE) acetabular cups (Hua et al.) as well review papers regarding the synergetic therapy combining lubrication and drug intervention, regulatory balance between bone resorption and formation, and exercise therapy (Ji and Zhang, 2019) and the latest technologies in the field of joint arthroplasty, such as osseointegration of artificial joints, the improvement of materials with the property of osteoimmunomodulation reviewing the improvement of

\section{REFERENCES}

Abdel-Jaber, S., Belvedere, C., Leardini, A., and Affatato, S. (2015). Wear simulation of total knee prostheses using load and kinematics waveforms from stair climbing. J. Biomech. 48, 3830-3836. doi: 10.1016/j.jbiomech.2015. 09.007

Affatato, S., Merola, M., and Ruggiero, A. (2018). Development of a novel in silico model to investigate the influence of radial clearance on the acetabular cup contact pressure in hip implants. Materials 11:1282. doi: 10.3390/ma11 081282

Affatato, S., Ruggiero, A., and Merolab, M. (2015). Advanced biomaterials in hip joint arthroplasty. A review on polymer and ceramics composites as alternative bearings. Compos Part B Eng. 83, 276-283. doi: 10.1016/j.compositesb.2015.07.019

Affatato, S., Ruggiero, R., Merola, M., and Logozzoc, S. (2017). Does metal transfer differ on retrieved Biolox $\AA$ Delta composites femoral heads? Surface investigation on three Biolox ${ }^{\circledR}$ generations from a biotribological point of view. Compos Part B Eng. 113, 164-173. doi: 10.1016/j.compositesb.2017.01.026

Dowson, D. (1970). Whither tribology? Wear 16, 303-304.

Ji, X., and Zhang, H. (2019). Current strategies for the treatment of early stage osteoarthritis. Front. Mech. Eng. 5:00057. doi: 10.3389/fmech.2019.00057

Jin, Z. M., Stone, M., Ingham, E., and Fisher, J. (2006). (v) Biotribology. Curr Orthopa. 20, 32-40. doi: 10.1016/j.cuor.2005.09.005

Kaddick, C., Catelas, I., Pennekamp, P. H., and Wimmer, M. A. (2009). Implant wear and aseptic loosening. An overview. Der. Orthopade. 38, 690-697. doi: 10.1007/s00132-009-1431-9

Kurtz, S., Ong, K., Lau, E., Mowat, F., and Halpern, M. (2007). Projections of primary and revision hip and knee arthroplasty in the United States from 2005 to 2030. J. Bone Jt. Surg. 89, 780-785. doi: 10.2106/JBJS.F.00222

Learmonth, I. D., Young, C., and Rorabeck, C. (2007). The operation of the century: total hip replacement. Lancet 370, 1508-1519. doi: 10.1016/S0140-6736(07)60457-7 joint arthroplasty technologies based on the modeling of dynamic osteosynthesis, as well as the identification of possible unconventional designs of artificial joints that contribute to these technologies, predictive assessment of areas for technologies improvement (Poliakov et al.). Moreover, attention was paid to review recent and relevant researches regarding the biotribo-corrosion phenomena in the oral rehabilitation devices summarizing clinical problems and advances obtained based on current scientific evidences in the framework of the tribological fundamentals, testing methodologies, and protocols in tribocorrosion analyses (Dini et al.).

We hope that the readers will find interesting insights in the published paper in this special issue and finally we would like to thank all authors for their valuable contributions, the involved and the Specialty Chief Editor(S) Roman Pohrt and Valentin L. Popov for their support during the entire process.

\section{AUTHOR CONTRIBUTIONS}

All authors listed have made a substantial, direct and intellectual contribution to the work, and approved it for publication.

\section{ACKNOWLEDGMENTS}

The authors acknowledge financial support of the Italian Research Project PRIN 2017 BIONIC-MIUR and National Natural Science Foundation of China (51675296).
Mattei, L., Di Puccio, F., Joyce, T. J., Ciulli, E., and Mattei, L. (2016). Effect of size and dimensional tolerance of reverse total shoulder arthroplasty on wear: an in-silico study. J. Mech. Behav. Biomed. Mater. 61:455-463. doi: 10.1016/j.jmbbm.2016.03.033

Merola, M., Ruggiero, A., De Mattia, S. J., and Affatato, S. (2016). On the tribological behavior of retrieved hip femoral heads affected by metallic debris. A comparative investigation by stylus and optical profilometer for a new roughness measurement protocol. Measurement 90, 365-371. doi: 10.1016/j.measurement.2016.05.003

O'Brien, S. T., Luo, Y., and Brandt, J. M. (2015). In-vitro and in-silico investigations on the influence of contact pressure on cross-linked polyethylene wear in total knee replacements. Wear 332, 687-693. doi: 10.1016/j.wear.2015. 02.048

Popov, V. L. (2010). Contact Mechanics and Friction. Berlin: Springer Berlin Heidelberg. doi: 10.1007/978-3-662-53081-8

Ruggiero, A., D, Amato, R., Gómez, E., and Merola, M. (2016). Experimental comparison on tribological pairs UHMWPE/TIAL6V4 alloy, UHMWPE/AISI316L austenitic stainless and UHMWPE/AL2O3 ceramic, under dry and lubricated conditions. Tribol. Int. 96, 349-360. doi: 10.1016/j.triboint.2015.12.041

Ruggiero, A., D'Amato R, and Affatato S. (2019). Comparison of meshing strategies in THR finite element modelling. Materials 12:2332. doi: 10.3390/ma121 42332

Ruggiero, A., Merola, M., and Affatato, S. (2018). Finite element simulations of hard-on-soft hip joint prosthesis accounting for dynamic loads calculated from a musculoskeletal model during walking. Materials 11:574. doi: 10.3390/ma11040574

Ruggiero, A., and Sicilia, A. (2020). Lubrication modeling and wear calculation in artificial hip joint during the gait. Tribol. Int. 142:105993. doi: 10.1016/j.triboint.2019.105993

Tan, S. C., Lau, A. C., Del Balso, C., Howard, J. L., Lanting, B. A., and Teeter, M. G. (2016). Tribocorrosion: ceramic and oxidized zirconium vs 
cobalt-chromium heads in total hip arthroplasty. J. Arthropl. 31, 2064-2071. doi: 10.1016/j.arth.2016.02.027

Ten Kate, J., Smit, G., and Breedveld, P. (2017). 3D-printed upper limb prostheses: a review. Disabil. Rehab. Assist. Technol. 12, 300-314. doi: 10.1080/17483107.2016.1253117

Vakis, A. I., Yastrebov, V. A., Scheibert, J., Nicola, L., Dini, D., Minfray, C., et al. (2018). Modeling and simulation in tribology across scales: an overview. Tribol. Int. 125, 169-199. doi: 10.1016/j.triboint.2018. 02.005

Zhang, J, Chen, Z., Wang, L., Li, D., and Jin, Z. (2017). A patient-specific wear prediction framework for an artificial knee joint with coupled musculoskeletal multibody-dynamics and finite element analysis. Tribol. Int. 109, 382-389. doi: 10.1016/j.triboint.2016.10.050

Zhongmin, J., Meng, Y., Hu, Y., and Luo, J. (2020). In memoriam: duncan dowson (1928-2020). Friction 8, 1-3. doi: 10.1007/s40544-020-0360-9
Zivic, F., Affatato, S., Trajanovic, M., Schnabelrauch, M., Grujovic, N., and Choy, K. L (Eds). (2017). Biomaterials in Clinical Practice: Advances in Clinical Research and Medical Devices. Springer. doi: 10.1007/978-3-319-68025-5

Conflict of Interest: The authors declare that the research was conducted in the absence of any commercial or financial relationships that could be construed as a potential conflict of interest.

Copyright $\odot 2020$ Ruggiero and Zhang. This is an open-access article distributed under the terms of the Creative Commons Attribution License (CC BY). The use, distribution or reproduction in other forums is permitted, provided the original author(s) and the copyright owner(s) are credited and that the original publication in this journal is cited, in accordance with accepted academic practice. No use, distribution or reproduction is permitted which does not comply with these terms. 